que goce de mi Celia los abrazos, de Celia, más hermosa que jazmín blanco y encarnada rosa.

Este amor, que había nacido ya hacía tiempo, no le impidió llorar con sincero dolor la muerte de la esposa. Lope era así: una pasión no excluía otro cariño. Y.después de todo, ¿no conocemos hombres de todos los tiempos que no han respetado la monogamia? El que se censure más a Lope estriba en que confiesa sus culpas a voces.

Es de suponer que Lope de Vega se estableciese en Madrid en el invierno de 1595 .

Quién era la Celia que le traía tan embelesado, será en breve objeto de un especial estudio.

Madrid.

María Goyri de Menéndez Pidal

\title{
MÚSICA, INSTRUMENTOS Y DANZAS EN LAS OBRAS DE GERVANTES
}

\section{ADICIONES I}

A LA PÁG. 23. - Sobre los romances y canciones en Cervantes, véase Miguel Querol, La música de los romances y canciones mencionados por Cervantes en sus obras, en AMIEM, I947.

A LA PÁG. 24. Tramoyas. - Alessandro d'Ancona, en Origini del teatro italiano (Torino, I89I), da noticias de la manera de representar en Francia los Misterios, y, en Italia, las Sacre Rappresentazioni. Infiernos y paraísos se describen minuciosamente en las instrucciones que se daban al efecto. Las desapariciones por medio de contrapesos se emplearon en Francia desde los primeros tiempos, y, en Italia, en piezas de teatro religioso popular como la representación de Santa Úrsula. "Si apre il Paradiso", se indica allí, lo cual se repite con alguna frecuencia en otras piezas. La tramoya se conocía en Francia como secrets o feyntes; en Italia, como ingegni. " $\mathrm{E}$ sebbene taluni affermino che eglino sono stati trovati molto prima, il Vasari segue la comune opinione, che Filipo Brunelleschi fosse l'inventore di quelli almeno che adornavano meravigliosamente il Paradiso della Rappresentazione dell'Annunziata, e de' quali il biografo aretino ci ha lasciato lunga e minuta descrizione". Descendía del cielo una mandorla con un ángel que saludaba a la Virgen, mientras lucían "infiniti lumi" y sonaban "dolcissime musiche". El cielo se abría y cerraba mediante el juego de las nubes, y ascendían las almas de los santos y otros bienaventurados. Desde entonces, las descripciones abundan, cada vez con mayor riqueza de ingeniosidades. Véanse, en el Libro segundo de la obra de Ancona, los capítulos virI (Asseto scenico della Sacra Rappresentazione), Ix (Glingeni teatrali), $\mathrm{x}$ (Intermezzi e pompe sceniche) y siguientes, que se refieren a los diferentes personajes que figuran en esta clase de teatro religioso-popular

1 Véase $N R F H$, II, 1948, págs. 21-70 y 118-1 73. 
medieval, en los albores ya del Renacimiento. En el Libro tercero, véase el capítulo I (Drammi profani del secolo xv e xvi modellati sulla Sacra Rappresentazione), así como los apéndices primero (La rappresentazione drammatica del contado toscano) y segundo (Il teatro mantovano nel secolo xvi).

A LA PÁG. $35^{-}$- Ciertos pasajes de Cervantes reflejan tópicos literarios muy divulgados en otros países, en Francia por lo menos, en tiempos anteriores a nuestro escritor. He aquí algunos casos. La "armonía" y "concertado canto" de los pajarillos se encuentra en citas que van desde la simple descripción poética en Gilles de Chin, siglo xiv, a las más imbuidas de sabiduría de la Messe des oiseaux de Bauduin de Condé. En Guillaume de Machaut ( I349) los pájaros cantan según las reglas del arte de esa época, en su "chapitre, tenu de sons et de hoquès" (el hoquet u ochetus, música dividida en varias voces, broken music en Bacon). En Les échecs amoureux el autor reconoce en la música pajaril un orden que estima ser natural de "l'art de musique et la mesure", y Cristina de Pisan (I400) piensa que esos cantores alados saben tanto como los chantres en sus disciplinas. En I 407 Eberhard Cersne, autor de Der Minne Regel, analiza muy seriamente el paralelismo entre la fogel musica y la que hacen los compositores más sabios del momento. André Pirro en su Histoire de la musique, $x v^{e}$ et $x v i^{e}$ siècles (Paris, I940, págs. 27 y sig.) hace otras menciones de Chaucer, Fadet Joglar, Giraud Riquier y Giraud de Calanson.

$\mathrm{El}$ "sosegado y maravilloso silencio" que guardan los pastores antes de que Orompo recite sus octavas era una buena costumbre que se recomienda en el roman de Pierceforest cuando una damisela va a mostrar sus proezas en el arpa, y cuando un heraldo llega para declamar un lai, acompañándose con el mismo instrumento. Cuando Minuccio canta en el Decamerón, acompañándose con la viola, todos los oyentes se quedan "taciti e sospesi ad ascoltare".

La especificación de las voces cantantes en "tiples, contras y tenores", o distinciones análogas que saben hacer hasta las gentes rústicas, aparecen frecuentemente en los poetas franceses del xII y del xIv, a veces con caracteres pedantescos, como en el poema sobre la caza de Gaces de la Buigne, en I 359, en el roman de Bauduin de Sebourc y en el Parfait du Paon, citas eruditas que tiempo después reaparecen, por ejemplo, en Fernán López de Yanguas, en Francisco de Avendaño, en Diego Sánchez de Badajoz, Antonio de Torquemada, f́nigo de Mendoza, Juan de Mena, Fernán Pérez de Guzmán, o en Gil Vicente, músico calificado. (Más ejemplos, en Francia, durante el xiv, en Pirro, ob. cit., págs. 35 y sigs.).

A LA PÁG. 37. Sobre la "guitarra española". - Algún autor (G. Kinskx, A history of music in pictures [Leipzig, I929], London, 1937, pág. I 40 , ej. I) dice que la guitarra española del siglo xviI se conocía en Alemania como quinterne, aludiendo quizá a sus cinco cuerdas. El nombre de quinterne nada tiene que ver en Alemania, ni en ninguna parte, con las cuerdas del instrumento: es simplemente adaptación del fr. guiterre-guiterne (en Mersenne; cf. CuRT SAcrs, Handbuch der 
Musikinstrumentenkunde, Leipzig, 1930, pág. 233); quintaria en el siglo xIv; quinterne en Cersne, op. cit.; guinterne y chiterne en Francia, a principios del siglo xv. Un grabado de Tobías Stimmer, en la primera mitad del xvi, muestra una quinterne alemana idéntica a la guitarra de Mersenne (véase pág. I 55), un siglo después, mencionada por este autor como instrumento antiguo, con cuatro órdenes de cuerdas, dobladas las tres más graves: $2+2+2+\mathrm{I}$. La quinterne de la lámina XVI, núm. 5, del Syntagma musicum de Michael Praetorius, $2^{a}$ parte (Wolfenbüttel, I6I8-16I9), consta de un cuerpo de guitarra, clavijero de tiorba adornado y seis cuerdas (cinco dobles y la prima sencilla) con ocho trastes.

Aunque Vicente Espinel no fuese el primero en añadir una quinta cuerda a la guitarra común, es útil recordar que las denominaciones de prima y quinta eran, en su época y hasta los tiempos de Mersenne, intercambiables entre los vocabularios francés e italiano. En el Livre second, Des instrumens, de su Harmonie universelle (Paris, I636), pág. 96, Mersenne dice: "La dernière chorde, c'est à dire la chanterelle, que les Italiens content la dernière, au lieu que nous la mettons la première ..." Y en la pág. 27 del Liber primus, De instrumentis harmonicis, de sus Harmonicorum libri XII (Paris, I648), reducción en lengua latina de la Harmonie universelle, hablando otra vez de la guitarra española (llamada por él cithara hispanica), dice refiriéndose a la cuerda más grave: "quae chordae Quintae tribuitur à nostris citaroedis, licet Itali primam numerent". En España, Venegas de Henestrosa, Libro de cifra nueva (Alcalá de Henares, I557), llama sexta a la prima de la vihuela.

A LA PÁG. 38, NOTA. - El pío pero ramplón coplero no inventaba nada al convertir la guitarra en una especie de potro donde se estiraban los nervios de Cristo, por semejanza a su suplicio en la cruz. Ya lo había pensado así Michel du Pressoir († I302) refiriéndose a las cuerdas del arpa (Pirro, ob. cit., pág. 53).

A LA PÁG. 39. - La trompeta de la fama suena también en la $\mathrm{Nu}$ mancia. El efecto exultante de la trompeta es tradicional. Cf. Eneida, lib. VI: "Aere ciere viros, martemque accendere cantus", y JuAN DE Padilla, Los doce triunfos, IV, cap. v: "Suena de pronto su ronca bocina / incitadora de bélica saña". Una acepción curiosa de lo bélico aparece en el Persiles (ed. de Madrid, I803, vol. II, pág. I43): "... fueron recebidos de los turcos con grande grita y algazara al son de muchas dulzainas y de otros instrumentos, que puesto que eran bélicos, eran regocijados"... Aquí puesto que es normal. Recuérdese que el ruido y el estrépito eran cualidades estéticas en tiempos anteriores a Cervantes. Así encontramos cómo se pondera en el Tirant lo Blanch "tanta remor e magnificencia" que hacían los "instrumentos altos" repartidos en las torres y ventanas de las salas, y en el Libro de Alexandre se decía ya que "el pleito de ioglares era fiera nota". En Francia, las citaciones abundan en este sentido. En Les échecs amoureux (hacia I370-80) se lee: "On sonnoit les haulz instrumens / Que mieulx aux danses plaisoient / Pour la grant noise qu'ils faisoient", y en el roman de Cléomades: "Plenté d'estrumens oyssiés... / Qui moult tres grant 
noise faisoient". Hay muchos ejemplos de estos deleitosos estrépitos, sin perjuicio de que la música dulce de "instrumens bas" o "instrumens coys" dejase de ser apreciada.

A LA PÁG. 40. Trompetas bastardas. - Las bastardas eran además piezas de artillería.

A LA PÁG. 46. - Esta instrumentación de Monipodio no era, con todo, invención suya. Ya la encontramos en frey Íñigo de Mendoza (Vita Christi, en el Cancionero de Foulché-Delbosc, vol. I, pág. r 8) :

$$
\begin{aligned}
& \text { y tienpla bien tu guitarra } \\
& \text { y yo con una piçarra } \\
& \text { començemos de baylar. } \\
& \text { Saquemos en cucharal } \\
& \text { y tan bien mi caramillo... }
\end{aligned}
$$

Estos versos pudieron parecer extraños o de un sentido incomprensible. Cuando se ve a los negros de Cuba acompañándose con cucharas, cajas de madera y pedazos de platos, a más de otros instrumentos como guitarras y cornetines, la orquesta pastoril de frey Íñigo no tendrá ningún misterio.

A LA PÁG. 49. - Al identificar Rodríguez Marín la "trompa de Paris", mencionada por Cervantes en el Celoso extremeño, con el birimbao o guimbarda se guió, probablemente, por la indicación de Mersenne (Harm. universelle, Livre septiesme, Des instrumens de percussion, pág. 49), el cual, al referirse al mencionado instrumento -que, bien puede decirse, es "unipersonal" -, lo denomina "Trompe, que quelquesuns nomment Gronde ou Rebube", y añade: "cet instrument sert aux laquais et aux gens de basse condition". (Por lo demás, su clasificación organográfica entra en el grupo de "láminas vibrantes"). En el siglo xvir se le denominaba en Francia "trompe d'Allemagne", según se lee en J. B. Weckerlin, Musiciana (París, I877, pág. Io8).

A LA PÁG. 53. - Se encuentra en frey Íñigo de Mendoza (Vita Christi, loc. cit., pág. 20): "lieuva tu el caramiello, / los albogues y el rabé" (para hacer "un huerte son agudiello" de carácter danzable). Parece que, cuando se trata de los albogues como instrumentos de percusión, se mencionan siempre en plural.

A rA PÁG. 54. Sobre la zampoña de alcacel. - El instrumentillo mencionado por la sobrina de don Quijote, que consiste en una cañita de cebada en que se hace una incisión de abajo arriba, de tal manera que quede libre una lengüecilla cuya vibración produce el sonido en el instrumento (al comunicarse a la columna interior de aire), es en Mersenne (Harm. univ.) una cañita de trigo, "tuyau de bled" (Libro quinto, págs. 282-283). Mersenne sigue a Lucrecio en su idea de que este instrumento fué el primero de viento conocido: "el susurro del céfiro en la oquedad de las cañas vacías enseñó a los rústicos a soplar en los tallos huecos" (De rerum natura, V, I 380 y sigs.). Mersenne se da cuenta perfecta de que el instrumento pertenece al tipo chalumeau-clarinete, y se expresa así (Proposition xxxv): "La chalemie ou la corne- 
muse rurale ou pastorale des bergers. Cet instrument est le premier de tous ceus qui usent d'anches... or ces anches se font de plusieurs matières, dont la plus simple est d'un chalumeau ou tuyau de bled". Una graciosa ilustración en que se ve a dos zagales tallando cañas para hacerse esas zampoñuelas se encuentra en el libro, en su tiempo famoso, de Filippo Buonanni (Bonanni) Gabinetto armonico pieno d'istromenti sonori (Roma, I 722), grabado xxvn, con la denominación explícita de Zampogne. Al apostillar Ceruti el libro de Bonanni (Descrizione degli istromenti d'ogni genere del padre Bonanni, seconda edizione riveduta, corretta e accesciuta dall'abate Giacinto Cervti ..., Roma, I776), utiliza los mismos grabados, originales de Arnoldo Wanwesterout.

A LA PÁG. 56. - Sobre la llamada Misa del asno y su relación con el "Office des fous", cf. H. Villetard, Office de Pierre de Corbeil (Office de la circoncision) improprement appelé Office des fous, texte et chant publiés d'après le Manuscrit de Sens (xII ${ }^{\mathrm{e}}$ siècle), Paris, Picard, I907.

A LA PÁG. I22. - La descripción que hace Mersenne de la zarabanda en su Harmonicorum libri XII (Liber secundus, De instrumentis pneumaticis, pág. 83) es interesante como testimonio no muy lejano de las prácticas españolas, $\mathrm{y}$ antes de que hubiese perdido su carácter tras de haberse convertido en una danza ceremoniosa en las piezas destinadas a la corte francesa. "Ubi notat - dice Mersenne - choraulem concinisse tibiis cantanti Symphoniae 7 [?] symphoniacis constanti, quanquam et de saltante ad caua buxa ${ }^{2}$, seu tibias intelligatur, solebat enim more Hispaniorum Sarabandam saltantium cheironomein, hoc est hue illue iactare manus, et tibiam, sed et podonomein, quod tanta dexteritate illi faciunt ut nescias quid magis in Hispano saltante et castaneolas digitis concutiente mireris, an capitis, an pedum, an digitorum, totiusque corporis ad numerum compositas agitationes, quas vix adaequent Neurobates licet carmen spiritu vibrantes". . .

A LA PÁG. I 30. - Sobre la morisca pueden encontrarse algunas referencias en mi libro La música en la sociedad europea, México, I944, vol. II, págs. 24, 90, I68. También en La danza y el ballet, México, I949, págs. 89-9o.

A la PáG. I 31 . - "Andar una danza" se lee en Juan de Padilla, Los doce triunfos, II, cap. xIv: "andar una danza de mala manera ..."

A LA PÁG. I4I. La Noche canta. - Una negra representaba la Noche en el "Ballet du triomphe de Minerva", i6 I5. (V. Prunières, Le ballet de cour en France, Paris, I9I4).

A LA PÁG. I 44. Viola-guitarra. — “También la guitarra, cuya descendencia dél fiedel medieval es fácil de demostrar, se denominó, en un principio, viola. Tinctoris, en su Ensayo de un diccionario de música (Terminorum musicae diffinitorium, Nápoles, hacia I474), considera la guitarra -ista vero plana - como una viola. En tiempos modernos aparece el nombre de "guitarra española" y asimismo el de "guitarra de

2 Flautillas de boj. 
cinco órdenes", en oposición a la guitarra corriente de cuatro órdenes, y, por último, el nombre de "guitarra", sin especificaciones. Este instrumento, hasta comienzos del siglo xviI, constituyó la hermana distinguida de la guitarra ordinaria; después, ésta desapareció por las mismas razones que el laúd. Ya en la Edad Media emigró a la península de los Apeninos. A fines del siglo xm, la Italia del sur estaba bajo el dominio de la corona de Aragón. Probablemente allí se la llamó viola. Los inventarios de la Casa de Este, por ejemplo, hablan de una "viola alia napoletana" en oposición a la "viola de arco". (CURT SAGHs, Handbuch der Musikinstrumentenkunde, Leipzig, I93o, págs. 2 I 2 y 230). Tinctoris, en la obra mencionada, asigna ya a la guitarra la calificación de "hispanorum invento". El vocablo castellano que corresponde, desde los tiempos más antiguos, al de viola es vihuela; la especificación de que se trata, en España, de vihuela punteada y no tocada con arco se hace, raras veces, denominándola "vihuela de mano"; pero como su práctica se extiende en ese país mucho más que la vihuela de arco, "vihuela", a secas, viene a ser la punteada, es decir, la violavihuela-guitarra, mientras que se prefiere especificar el otro modo de juego diciendo "vihuela de arco". En Italia, la difusión principal se hizo a favor de este instrumento. Sin embargo, no se especificó el punteado diciendo "viola da mano", sino que a la de arco se la denominó "viola da braccio" que, en los tiempos más tempranos, supone esta diferenciación y no sólo el tamaño menor que las violas "da spalla" o "da gamba". Un ejemplar curioso, y tardío, porque es de fines del siglo xvn, es el inventariado con el número I047 en la colección de instrumentos musicales del Metropolitan Museum de Nueva York. Se trata de una viola-guitarra construida en el Tirol (Pat. Johannes fec. in Apsum, I688), cuyo cuerpo muestra ser el de una viola de transición, según comienza a verse en grabados alemanes del siglo xvi (Triunfo de Maximiliano) y flamencos del xvn. La parte superior de las escotaduras se talla en bisel o ángulo, mientras que la inferior resbala en curva. Es lo contrario de lo que ocurre en los primeros casos de las violas italianas, que conducen al tipo de viola-violín, tras de la doble escotadura (éclisse) lisa, que conservan las guitarras. Esta viola-guitarra tiene un clavijero plano, en forma de ocho, con las clavijas insertas en la parte posterior, muy popularizado en los países meridionales. Las cuerdas son seis, con doce trastes, los tres agudos para posiciones poco usadas. La mitad superior del cuerpo es más pequeña que la inferior. La rosa se sitúa en la confluencia de ambas partes. El cordal es plano.

A LA PÁG. I47, línea I6: léase sexta cuerda; línea I8: léase siglo xviii.

A LA PÁG. I53. Guitarras de seis y siete cuerdas en los siglos xvi y xvii. - Contra lo que se suele decir acerca de la añadidura de la cuerda grave a las guitarras de cinco cuerdas, en el siglo xvnt, se encuentran testimonios de ellas en épocas muy anteriores, bien porque sean simplemente vihuelas o porque fueran guitarras españolas con la cuerda grave añadida ya en esas épocas. En la portada del libro de Bernhard Schmid, el Viejo (grabada por Tobias Stimmer), Einer neuen Kuenstli- 
chen Tablatur auf Orgel und Instrumenten, Estrasburgo, I 577, parece que se ven guitarras de seis cuerdas. La distribución de ellas en el dibujo podría responder a una guitarra de cuatro cuerdas con la primera y segunda dobladas, lo cual es anormal. Dada la índole del libro, debe tratarse de vihuelas, o de guitarras de seis cuerdas. El caso resulta igualmente interesante, sea como fuere. Hay otro ejemplo en el libro de Jacobo Paix Orgeltabulaturbuch, de I $^{83}$. En los dos dibujos, el clavijero tiene forma de ave. El grabado de Tobías Stimmer aparece ya en otro tratado alemán de laúd de 1572 (cf. G. KINSKy, ob. cit., pág. I 34, núm. I ). Otra guitarra de cuatro cuerdas en Alemania, siglo xvI, en manos de persona distinguida (dobladas: $2+2+2+1$ ) en la misma obra (pág. 96, núm. 5). Guitarras con seis cuerdas en los Países Bajos, en el siglo xvn, pueden verse en algunas pinturas como la de González Coques, en el Museo de Bruselas (seis cuerdas dobles) y, sobre todo, la que un tañedor y cantor toca en la "Saengergesellschaft" de Th. Rombouts (I597-I687). Es una guitarra enteramente del tipo español, con el clavijero en trapecio alargado y clavijas insertas en la parte posterior. Tiene siete órdenes de cuerdas; dobladas cuatro interiores, y simples la más grave, la prima y la segunda, como es fácil ver, sobre todo a la altura de ios dedos del ejecutante; pero solamente tiene seis trastes. El total de once cuerdas se confirma en el número de clavijas utilizadas (algunos orificios del clavijero, que habrían correspondido a clavijas para doblar más cuerdas, quedan vacíos). Sobre las vihuelas de siete cuerdas en España, véase el texto; y, en Italia, véase la ilustración de la pág. I 55). El cuadro de Rombouts se conserva en las Bayerische Staatsgemäldesammlungen, de Munich.

A LA PÁG. I54. Sobre las cuerdas y sus particularidades. - He podido reunir una pequeña bibliografía referente a estudios sobre las cuerdas de los instrumentos. En la mayor parte de los casos, los autores tratan teóricamente de las relaciones numéricas entre su longitud y grosor y el sonido que producen. Algunos, como Mersenne en su doble obra, en lengua francesa y latina, hablan de la fabricación de cuerdas metálicas; pero, a veces, una simple observación de pasada nos ilustra más sobre las cualidades de las cuerdas y sus posibilidades prácticas; por ejemplo, cuando Mersenne dice que, frecuentemente, la mitad inferior de ellas produce entonaciones falsas, lo cual explica algunas razones acerca de la inutilidad de prolongarlas demasiado y de emplear trastes en posiciones muy agudas. Fuera de la Harmonie universelle y de los Harmonicorum libri XII de Marin Mersenne (Paris, I636 y I648), las referencias que puedo ofrecer al lector son las siguientes:

I) L. CARRé, Traité mathématique des cordes par rapport aux instruments de musique. (En Mémoires de l'Académie des Sciences, $\mathrm{Pa}$ ris, I 706 , I 24 págs.).

2) Grande Encyclopédie (de Diderot y d'Alembert). Artículo Boyaudier (Paris, I 759). El artículo fué suprimido en la Encyclopédie Méthodique de Framery et Guinguené, tomo I, Paris, I79I, que reproduce los de J. J. Rousseau en la obra mencionada más abajo; pero como éste copia en parte las observaciones de d'Alembert pertenecientes a aquella otra obra, la nueva enciclopedia los inserta a su vez. 
3) Giovanni Sacahi, Del numero e delle misure delle corde musiche e loro corrispondenze, Milano, I $76 \mathrm{I}$.

4) J. J. Rousseau, Dictionnaire de Musique (terminado en I750; dado a imprimir en $1764-1765)$. Al reproducir sus artículos sobre las cuerdas la Encyclopédie Méthodique de Framery et Guinguené lo hace con las observaciones de M. Suremain de Missery y M. l'Abbé Feyton, que corrigen algunas afirmaciones de Rousseau.

5) J. J. DE Lalande, Voyage d'un Français en Italie dans les années 1765 et 1766 , Venecia, I 769, vol. VI, págs. 407-4I4.

6) M. Delezenne, Membre résident, Expériences et observations sur les cordes des instruments à archet, sin fecha, i 8 ... I I 4 págs.

7) C. BAud, Observations sur les cordes à instruments' de musique, Versailles, I 803, 47 págs. ( I 2 Thermidor, año Io). El autor dirige su escrito a Gossec defendiendo la excelencia de las cuerdas de seda y Gossec responde aprobando la tesis de Baud, y diciendo, además, que los chinos y los griegos conocían esta clase de cuerdas. Puede despren-

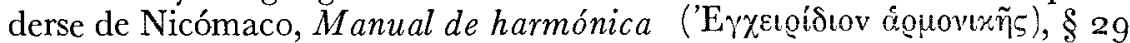
(Meibom, pág. I I) que los griegos, a lo menos en su tiempo (mediados del siglo II d. J. C.), fabricaban sus cuerdas retorciendo hilos simples, pero no sabemos de qué materia eran. El número de estos hilos proporcionaba el tono deseado como fundamental de la cuerda, por ejemplo, una cuerda de dos hilos daba la octava inferior a una cuerda de un hilo solo.

8) M. Plassiard, Des cordes du violon (en Association française pour l'avancement des Sciences, Congrès de Lille, I 874 . I92 págs.).

Del número 3 se encuentra un ejemplar en la Biblioteca Nazionale, de Turin; de los números 6 y 7 los hay en la Widener Library, Harvard University, Cambridge, Mass., donde he podido consultarlos. Los números I, 2, 5 y 8 me han sido comunicados por M. Marc Pincherle, Presidente de la Société Française de Musicologie, París.

A LA PÁG. I 55. - Mersenne abandona el clavijero curvo con cajuela que muestra en la guitarra publicada en la pág. 95 del Livre second, Des instrumens, en su Harmonie universelle (reproducido en nuestra pág. I 55, reminiscente todavía de los clavijeros de las cetrasviolas), por el más común, plano, con las clavijas insertas en la parte posterior, generalizado en la "guitarra española", cuando muestra este instrumento ("Cithara Hispanica") en sus Harmonicorum libri XII, posterior en doce años a aquel otro ingente tratado (pág. 27 del Liber primus, De instrumentis harmonicis). Esas volutas del clavijero, dice, no son necesarias ("binas volutas tametsi non necessarias habet"), por lo cual su sustitución por el clavijero plano no presenta inconvenientes. El doblaje de las cuerdas es, a primera vista, un poco desconcertante, porque resulta que las dos primeras cuerdas (del grave al agudo) están más altas, en la descripción de Mersenne, que la tercera ${ }^{3}$; es decir, que la afinación que muestra en la pág. 96 (junto a una guitarra más moderna con clavijero plano) resultaría, prescindiendo del lugar de las cuerdas en ella, en este orden: fa sol la do re, lo cual no tiene sentido.

${ }^{3}$ Sin embargo, en la pág. 28 dice taxativamente: "atque adeo gravior est prima". 
La razón parece ser la siguiente. Las dos cuerdas graves, sol do, más altas que la tercera, proceden de la duplicación a la octava aguda de las fundamentales; pero estos sonidos tenían, para el autor, un timbre desagradable ("ces sons tiennent quelque chose du chaudron"), por lo cual renuncia a la duplicación en octavas y se atiene a la nota alta de esa duplicación, poniendo ambas cuerdas al unísono ("quorum unusquisque duos nervos aequales, et unísonos"). El resultado es anómalo, y Mersenne lo reconoce diciendo que "est particulier à l'accord de la Guiterre". Una vez que se obtuvieron mejores cuerdas graves, se renunció al doblaje, y la anomalía (si es que estaba generalizada, y parece que no lo estaba) desapareció. Las tres guitarras que Mersenne muestra en sus dos libros, tanto la más antigua como las más modernas, tienen siempre ocho trastes, aunque en el tasto quede espacio libre para más; pero ya se ha indicado por qué Mersenne evita emplear la mitad inferior de las cuerdas.

A LA PÁG. 157. La afinación de la guitarra en Mersenne. - El lector desprevenido, no habituado a las complicaciones y enredos de la teoría antigua, podría encontrar que existe una incompatibilidad entre lo explicado anteriormente sobre la afinación de las vihuelas en el siglo xvi y la que Mersenne adjudica a la guitarra en el xvn. Prescindiendo de la diferencia de circunstancias organográficas en una y otra época, puede verse que tal incongruencia es más aparente que real. El temple que Mersenne indica en el grabado de la pág. 95, Libro segundo de la Harmonie universelle antes aludido, es el normal por Sol: sol do fa la re, y así lo repite en sus Harmonicorum libri XII: "prima incipit G re", etc. Pero cuando traduce los signos de la página mencionada (en clave de sol) y los muestra en clave de fa en tercera, les da la denominación de re sol do mi la. Hemos encontrado esta afinación, en circunstancias especiales, en Gaspar Sanz (pág. 157, línea I I de la nota), y ésa era la afinación de los instrumentos agudos del tipo viola (en Martín Agrícola, I 528, y Silvestro Ganassi dal Fontego, I 542), lo cual era practicable en instrumentos de arco mejor que en los de pulso; a pesar de ello Hans Gerle (1532) recomienda la afinación por La, o sea una cuarta más baja. Pero no debe olvidarse lo anteriormente explicado acerca de la incertidumbre del diapasón en este tiempo (véase pág. I48). El temple re sol do mi la, que indica Mersenne, es puramente teórico, y no responde a una afinación real sino que lo adopta como el más fácil para mostrar los "nombres harmoniques" (frecuencias), que eran la gran especialidad de Mersenne. A fin de exponerlos en sus cifras más sencillas ("il n'en peut prendre de moindres"), toma esas notas, que responden a las frecuencias $27,3^{6,24}, 3^{\circ}, 4^{\circ}$, aunque claramente se ve que no convienen a la guitarra, pues que la más baja en sus mediciones, el Do 24, es la nota que corresponde a la cuerda grave del violoncelo. Pero las frecuencias indicadas corresponden a nuestros sonidos la (27), re (36), sol (24), si (30), mi (40). (Cf. Sir James Jeans, Science and music, Cambridge, I947, pág. 27), o más exactamente la $\left(27.5^{\circ}\right)$, re (36.7 I), sol (24.50), etc. (Cf. Zamminer, Die Musik und die musikalischen Instrumente, Giessen, I855; Rayleigh, The theory of sound, New York, $2^{\mathrm{a}}$ ed., 1945). 
A LA PÁG. I58.- La pieza titulada $H e$ en el manuscrito I 4 I debe ser la que autores franceses e ingleses del siglo xvi mencionan como danza titulada Hay o Haye (Peter Warlock, Prefacio a la Orchesographie de Thoinot Arbeau, pág. I5 de la traducción castellana, Buenos Aires, 1946).

A LA PÁG. I 70.-Añádase a la bibliografía: Marius Schneider, $L a$ danza de espadas y la tarantela, Instituto Español de Musicología, Barcelona, i948.

Al cuadro, fuera de Texto (frente a la pág. i6o). - La idea de que cetra y cítola fueron en su origen instrumentos distintos, derivado el primero de la gran rama de la kythara y el segundo de la fythelefidula medieval, está apuntada por Curt Sachs en su Handbuch der Musikinstrumentenkunde. En la pág. 209 dice: "En los comienzos de la Edad Media son innumerables las menciones de las Fiedeln tañidas con los dedos (gezupften) ... Más tarde se levantan dos grupos: el primero deriva directamente del latín cithara, it. cetra, esp. cedra, fr. cithre, ing. cittern; el segundo, con finales en diminutivo: it. cetola, esp. citola, fr. citole, cuitole, med. a. al. zitôle, cistôle; med. b. al. sitole". Que las dos ramas cythara y fitola estaban aún claramente separadas en el siglo xnr se deduce de los comentarios a Alanus de Insulis, De planctu naturae: "Lira est quoddam genus cytharae vel fitola, alioquin de roet". Cuando en el xIv aparece la giterne-ghisterne, en las citas que se mencionan en el cuadro fuera de texto, es fácil ver que lo hacen con referencia a un entronque diferente de la fitheln, y que, por lo tanto, la ghisterne no se confunde con la citole (Les échecs amoureux). Ambas están también claramente distinguidas en el Ars Musica de Juan Egidio de Zamora (siglo XII) : "ut patet in viella et cythara et organis" (GERBERT, Scriptores, tomo II, pág. 378), "ut citharae ac viellae" (IDEM). Si es posible admitir dos acentuaciones, una grave para fidula, fitola y otra esdrújula, fidula, fítola, la primera en los países del norte, y la segunda en el sur, cabría pensar que la primera habría conducido a fythéle, viedel, fiedel, y de aquí a vièle, vielle, viola, mientras que la segunda habría llegado rápidamente a cétola, citola. La identificación posterior entre cedras y citolas vendría por semejanza en la función organográfica.

Sobre la gaita zamorana. -- En el Museo Instrumental del Conservatorio de Bruselas (Catalogue descriptif et analytique por V. Ch. Mahillon, tomo I, Gand, I 893) se encuentra una colección de instrumentos árabes procedentes de Egipto cuyas denominaciones se relacionan directamente con lo dicho en el cuadro fuera de texto acerca de la gaita zamorana. Esos instrumentos son de dos especies: de caña doble, afín al óboe, que es el zamr aludido en el texto (o Zourna, cuyas ilustraciones dejan ver su parecido con la dulzaina castellana), y los de caña simple, afines al clarinete, y que se denominan zummârah. Los instrumentos de caña simple denominados gheteh podrían ser asimilados al español "gaita" y los zummârah a la supuesta localización "zamorana".

La simultaneidad de instrumentos de caña simple y doble confirma su antigüedad en los estudios arqueológicos recientemente hechos acerca del aulós griego. Véase N. B. Bodley, The auloi of Meroe (American 
Journal of Archeology, abril-junio de 1946): instrumentos de las dos especies encontrados (en fragmentos) en I92 I en Méroe, Sudán, e importados, al parecer, por Alejandría en los primeros años de la era cristiana, o construidos en dicha ciudad (bibliografía sobre el aulós en el mismo trabajo).

Mersenne, en las obras reiteradamente citadas, analiza cuidadosamente los instrumentos "pneumáticos" de caña sencilla y doble. A los primeros les reconoce su origen en el chalumeau y con este nombre describe el tubo de la gaita y de la museta con embocadura de caña simple, lo cual extiende a la gaita entera, llamándola "psaltoritium utrem, quem Galli Chalemie vocant" (Harmonicorum libri XII. Líber secundus, De instrumentis, pág. 9I). Quizá esto explica la confusión de Laborde.

Corrijase en la nota 5 del mismo cuadro: zanfona (Vieja Castilla)... Prov. phonphogne.

Adolfo Salazar

E1 Colegio de México. 